\title{
Power-law electrokinetic behavior as a direct probe of effective surface viscosity
}

\author{
Yuki Uematsu ${ }^{\mathrm{a}, \mathrm{b}, *}$, Roland R. Netz ${ }^{\mathrm{b}}$, Douwe Jan Bonthuis ${ }^{\mathrm{b}}$ \\ ${ }^{a}$ Department of Chemistry, Kyushu University, Fukuoka 819-0395, Japan \\ ${ }^{b}$ Fachbereich Physik, Freie Universität Berlin, 14195 Berlin, Germany
}

\begin{abstract}
An exact solution to the Poisson-Boltzmann and Stokes equations is derived to describe the electric double layer with inhomogeneous dielectric and viscosity profiles in a lateral electric field. In the limit of strongly charged surfaces and low salinity, the electrokinetic flow magnitude follows a power law as a function of surface charge density. Remarkably, its exponent is determined by the interfacial dielectric constant and viscosity, the latter of which has eluded experimental determination. This provides a novel method to extract the effective interfacial viscosity from standard electrokinetic experiments. We find good agreement between our theory and experimental data.
\end{abstract}

Keywords: Electrokinetics, interfacial slip, liquid-solid interface, electric double layer

Introduction. - Electro-osmosis is the motion of an electrolyte solution induced by an electric field along a surface [1, 2, 3, 4, 5, 6, 7, 8, 9, 10, 11, 12], whereas electrophoresis is the electric-field-induced motion of a colloid suspended in an electrolyte $[1,2,3,13,14,15]$. These electrokinetic effects play important roles in industry and bio-systems $[16,17,18]$. Originating in the nanometer-wide electric double layer, both effects depend sensitively on the structure and the dynamic properties of this interfacial layer. Simulation studies demonstrate that the surface layer exhibits an increased viscosity at hydrophilic surfaces [19], in agreement with early theories used to explain electrokinetic experiments [3]. At hydrophobic surfaces, however, a

\footnotetext{
${ }^{*}$ Corresponding author

Email address: uematsu@chem.kyushu-univ.jp (Yuki Uematsu)
} 
depletion layer and a finite slip length are observed, indicating a decreased effective viscosity $[4,5,6,7,8,9,19,20,21]$. The interfacial dielectric profile of water has been studied using molecular dynamics (MD) simulations $[4,5,6,7,8,19,20,21,22,23,24,25,26]$, revealing a highly inhomogeneous and oscillating profile near the interface [22, 23]. Whereas an effective interfacial dielectric constant can be readily obtained from capacitance measurements, experimental determination of the interfacial viscosity has been controversial [27, 28, 29].

The traditional model describing electro-osmosis and electrophoresis is based on the Poisson-Boltzmann (PB) and Stokes equations [1, 2, 3]. However, this model fails to capture many experimental observations, such as the saturation of the electrokinetic flow with rising surface charge density $[3,30,31,32]$. The discrepancies have largely been resolved by computing numerical solutions to the PB and Stokes equations, including the dielectric and viscosity profiles extracted from simulations [24, 25]. Yet the numerical solutions cannot be used to unequivocally extract the interfacial profiles from experimental data without prior knowledge.

In this Letter, we construct an exact solution and derive analytical asymptotic expressions for the electrokinetic flow beyond the linear theory [33]. With these expressions, information about the subnanometer-scale interfacial properties can be directly acquired from standard electrokinetic experiments. In particular, in the limit of strongly charged surfaces and low salinity, we obtain an analytical power-law relation for the magnitude of the electrokinetic flow.

Central result. - We model the interfacial structure by a layer of width $z^{*}$, which has a dielectric constant $\varepsilon_{\text {int }}$ and viscosity $\eta_{\text {int }}$ different from the bulk values $\varepsilon$ and $\eta$. In addition, we include specific adsorption of monovalent ions into the interfacial layer, expressed by a constant chemical potential $k_{\mathrm{B}} T \alpha$ with $k_{\mathrm{B}} T$ being the thermal energy. Using this box model, we derive from the PB and Stokes equations (explained below) that in the limit of strongly charged surfaces and low salinity, the electrokinetic surface charge density $\sigma_{\text {ek }}$ scales with the bare surface charge density $\sigma_{0}$ as

$$
\left|\sigma_{\mathrm{ek}}\right| \approx \sqrt{\frac{\mathrm{e}^{\alpha} \varepsilon}{\varepsilon_{\mathrm{int}}}}\left(\frac{2 \varepsilon_{\mathrm{int}} \varepsilon_{0} k_{\mathrm{B}} T \operatorname{arccosh} \sqrt{\mathrm{e}^{\alpha} \varepsilon / \varepsilon_{\mathrm{int}}}}{e z^{*} \sqrt{\mathrm{e}^{\alpha} \varepsilon / \varepsilon_{\mathrm{int}}-1}}\right)^{1-\gamma}\left|\sigma_{0}\right|^{\gamma},
$$

with $\varepsilon_{0}$ being the electric permittivity of vacuum and $e$ the elementary charge. Notably, Eq. (1) exhibits a power-law behavior with exponent $\gamma \equiv \varepsilon_{\text {int }} \eta / \varepsilon \eta_{\text {int }}$. 

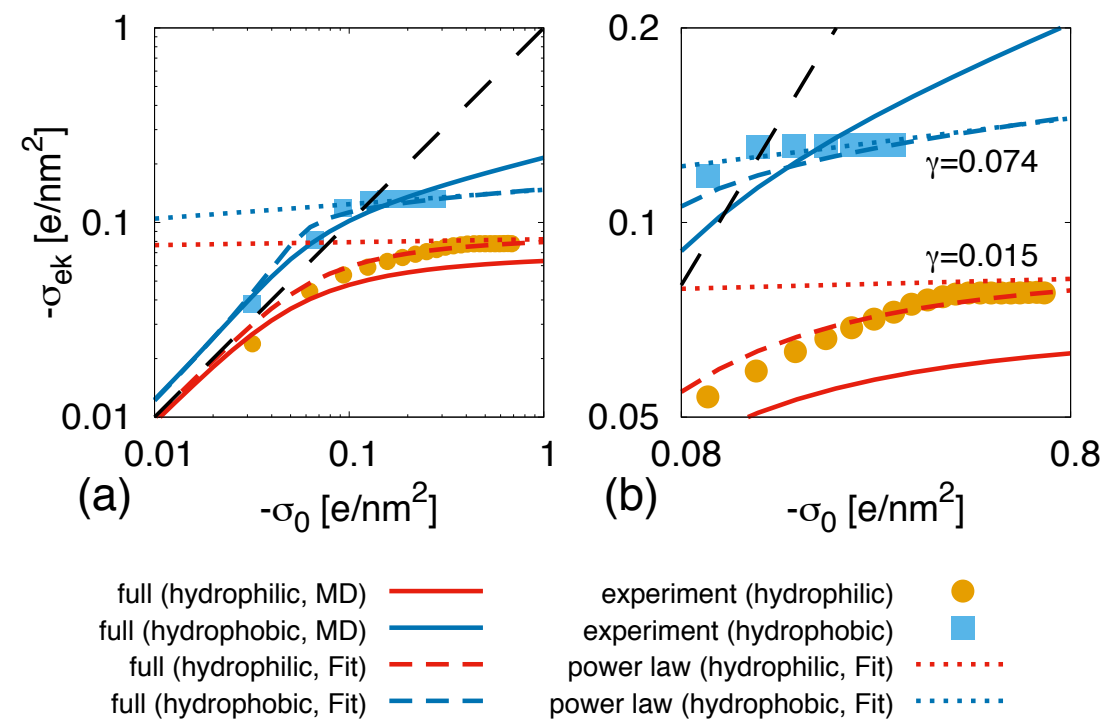

Figure 1: The electrokinetic surface charge density $\sigma_{\text {ek }}$ for a $c_{0}=1 \mathrm{mM}$ electrolyte solution as a function of the bare surface charge density $\sigma_{0}$ (double-logarithmic). The colored lines show the full analytical solutions [Eqs. (7), (8), (11), and (12)] with parameters obtained in MD simulations (solid lines, denoted MD) and with fitted parameters (broken lines, denoted Fit). The colored dotted lines denote Eq. (1) with fitted parameters. The black broken line denotes $\sigma_{\text {ek }}=\sigma_{0}$. We set $\varepsilon=78$ [35] and $T=298 \mathrm{~K}$. The interfacial parameters are summarized in Table 1 . Because the experimental data have been published as continuous lines [31], we have extracted data points every $0.5 \mu \mathrm{C} / \mathrm{cm}^{2}\left(\approx 0.03 e / \mathrm{nm}^{2}\right)$ for $\sigma_{0}<0$ (symbols). In order to cancel the surface conduction correction [31], the hydrophilic data have been reconstructed according to $\sigma_{\text {ek }}=\sinh \left(\operatorname{arcsinh}\left(\sigma_{\text {ek }}^{\text {corr }}\right) / 1.55\right)$, with $\sigma_{\mathrm{ek}}^{\text {corr }}$ corresponding to the value reported in reference [31]. (b) is a magnification of (a).

Fitting Eq. (1) to experimental data uniquely determines the equation's prefactor, as well as the exponent $\gamma$. Because $\varepsilon_{\text {int }}$ can be derived from capacitance measurements $[22,34]$, the procedure yields the interfacial viscosity $\eta_{\text {int }}$.

Fig. 1 shows a double-logarithmic plot of $\sigma_{\text {ek }}$. The experimental data (symbols) have been obtained with $\mathrm{TiO}_{2}$ (hydrophilic) and AgI (hydrophobic) in $\mathrm{KNO}_{3}$ aqueous solutions (explained in the caption of Fig. 1) [31]. Without interfacial effects $\sigma_{\mathrm{ek}}=\sigma_{0}$ (black broken line). The deviation of the data from the black broken line reveals the importance of the interfacial effects. The colored solid and broken lines denote the solutions defined by Eqs. (7) and (8) (see below), and the colored dotted lines denote Eq. (1). The interfacial parameters of the model are obtained in two different ways. First, 
we take the parameters obtained from MD simulations of generic hydrophilic and hydrophobic solid planar surfaces, which have been independently verified by comparison with multiple sets of experimental data on surface capacitance [22, 23], surface conduction and electrokinetics [24, 25]. Even though the simulations were performed for diamond surfaces instead of the surface types used in the experiments that we compare with, the advantage of this approach is the total absence of fit parameters. The specific adsorption parameter $\alpha$ is expected to depend sensitively on the combination of ions and surfaces used, and is set to $\alpha=0$ due to a lack of literature data. For hydrophilic surfaces, the slip length $b$ and $\eta_{\text {int }}$ are measurable in MD simulations [19]. Therefore, we use $z^{*}=b\left(\eta / \eta_{\text {int }}-1\right)^{-1}$, and $\varepsilon_{\text {int }}$ is determined by the value of the surface capacitance $\varepsilon_{\text {int }} / z^{*}[22,23,24,25]$. For hydrophobic surfaces, slip is caused by a low-density depletion layer, which can be modeled by a slip boundary condition or by a low-viscosity layer. Either description constitutes an approximation that captures the large-scale hydrodynamic properties well, but at the molecular scale, both approximations are imperfect. We choose $\varepsilon_{\text {int }}=1$, determining $z^{*}$ through the capacitance, and determine $\eta_{\text {int }}$ from $b$ by $\eta_{\text {int }}=\eta z^{*} /\left(b+z^{*}\right)$. For both surface types we observe a fair agreement between the experimental data and our analytical theory without fit parameters (Fig. 1, solid lines). However, this procedure without fit parameters predicts a higher slope than the one exhibited by the experimental data for hydrophobic surfaces (blue squares).

As a second approach, we extract the interfacial properties from a fit to the experimental data. At the hydrophilic surface, the exponent $\gamma$ extracted from the MD simulations provides a good fit to the experimental data (Fig. 1, dotted lines). Excellent agreement is achieved by fitting only $\alpha$ (Fig. 1, broken lines), resulting in the moderate value of $\alpha=1.1$. At the hydrophobic surface, we fit Eq. 1 to the high- $\sigma_{0}$ part of the curve shown in Fig. 1(b). As the quality of the decades-old data does not allow for a 4-parameter fit, we fix $\varepsilon_{\text {int }}$ and fit $z^{*}, \eta_{\text {int }} / \eta$ and $\alpha$. Good agreement with the experimental data is achieved using a $3.2 \AA$-wide low-viscosity layer (resulting slip length $b=1.6 \mathrm{~nm}$ ). The differences between the MD and fit parameters are not unexpected, given that the MD simulations where performed for diamond and the experiments for AgI. The stronger repulsion of ions from the interfacial layer at hydrophobic surfaces $(\alpha=10)$ than at hydrophilic surfaces $(\alpha=1.1)$ is consistent with ionic potentials of mean force calculated by MD simulations of hydrophobic and hydrophilic self-assembled monolayers [25]. The fitted and MD parameters used in Fig. 1 are summarized in Table 1. 
Table 1: Interfacial parameters from molecular dynamics simulations (MD) [19, 22] and from fitting (Fit). The interfacial capacitance $\varepsilon_{\text {int }} / z^{*}$ and the slip length $b$ are shown for comparing the difference between MD and Fit.

\begin{tabular}{cccccccc}
\hline & & $\varepsilon_{\text {int }}$ & $\begin{array}{c}z^{*} \\
(\mathrm{~nm})\end{array}$ & $\eta_{\text {int }} / \eta$ & $\alpha$ & $\begin{array}{c}\varepsilon_{\text {int }} / z^{*} \\
\left(\mathrm{~nm}^{-1}\right)\end{array}$ & $\begin{array}{c}b \\
(\mathrm{~nm})\end{array}$ \\
\hline hydrophilic & MD & 4.4 & 0.44 & $3.7[19]$ & 0.0 & $10[22]$ & $-0.32[19]$ \\
& Fit & 4.4 & 0.44 & 3.7 & 1.1 & 10 & -0.32 \\
\hline hydrophobic & MD & 1.0 & 0.12 & 0.054 & 0.0 & $8.3[22]$ & $2.1[19]$ \\
& Fit & 1.0 & 0.32 & 0.17 & 10 & 3.1 & 1.6 \\
\hline
\end{tabular}

Our theory and the comparison with experimental data reveal that the electrokinetic surface charge $\sigma_{\text {ek }}$ exhibits a power law behavior with a small but non-zero exponent ( $\gamma=0.074$ for the hydrophobic and $\gamma=0.015$ for the hydrophilic surface), determined by the subnanometer-wide interfacial structure. The fitting procedure works particularly well for hydrophilic surfaces, confirming the value of $\eta_{\text {int }}$ derived from MD simulations. Note that charged hydrophilic surfaces are also the most relevant in biological and industrial applications. Therefore, the newly-derived Eq. (1) provides a method to measure the parameters characterizing the interfacial structure, and in particular the elusive interfacial viscosity, by standard electrokinetic experiments.

Model. - To derive Eq. (1) we consider a planar interface, so that the electrostatic potential and all density distributions only depend on the perpendicular coordinate $z$. In the presence of an inhomogeneous local dielectric profile, the Poisson equation reads [22, 23]

$$
\frac{d}{d z}\left[\varepsilon_{\perp}(z) \varepsilon_{0} \frac{d}{d z} \psi(z)\right]=-\rho(z)
$$

where $\varepsilon_{0}$ is the vacuum permittivity, $\psi$ is the electrostatic potential, and $\rho$ is the ionic charge density. The dielectric profile $\varepsilon_{\perp}(z)$, calculated using MD simulations, shows strong oscillations [22, 23], but can be cast into a box profile $[22,23,24,25,33]$ to make the model analytically tractable,

$$
\varepsilon_{\perp}(z)= \begin{cases}\varepsilon_{\text {int }} & \text { for } 0<z<z^{*} \\ \varepsilon & \text { for } z>z^{*}\end{cases}
$$

where $\varepsilon_{\text {int }}$ is the interfacial dielectric constant, $\varepsilon$ is the dielectric constant of bulk water, and $z^{*}$ is the width of the interfacial layer [see Fig. 2(a)]. 
The charge density equals $\rho(z)=e\left[c_{+}(z)-c_{-}(z)\right]$, where $e$ is the elementary charge, and $c_{+}\left(c_{-}\right)$is the concentration of cations (anions). Neglecting steric ion effect [36, 37, 38, 39], we model monovalent ions whose concentrations obey a Boltzmann distribution, $c_{ \pm}(z)=c_{0} \exp \left[\mp \Psi(z)-\mu_{ \pm}(z)\right]$, where $c_{0}$ is the bulk salt concentration and $\Psi=e \psi / k_{\mathrm{B}} T$. Furthermore, $\mu_{+}(z)$ and $\mu_{-}(z)$ are non-electrostatic contributions to the potential, for which we use a box profile, $\mu_{ \pm}(z)=\alpha \theta\left(z^{*}-z\right)$, where $\alpha$ is an adjustable parameter and $\theta(z)$ is the Heaviside function [see Fig. 2(a)]. The boundary condition on the surface reads $d \psi /\left.d z\right|_{z=0}=-\sigma_{0} / \varepsilon_{\text {int }} \varepsilon_{0}$, where $\sigma_{0}$ is the bare surface charge density, and far away from the surface $\left.\psi\right|_{z \rightarrow \infty}=0$. At the boundary between the interfacial and the diffuse layer, we use continuity of the potential $\left.\psi(z)\right|_{z=z^{*}-0}=\left.\psi(z)\right|_{z=z^{*}+0}$, and continuity of the displacement field $\varepsilon_{\text {int }} d \psi /\left.d z\right|_{z=z^{*}-0}=\varepsilon d \psi /\left.d z\right|_{z=z^{*}+0}$. For an inhomogeneous viscosity profile, the hydrodynamics equation reads $[24,25]$

$$
\frac{d}{d z}\left[\eta_{\perp}(z) \frac{d u}{d z}\right]+\rho(z) E_{\|}=0,
$$

where $\eta_{\perp}(z)$ is the viscosity profile, $u(z)$ is the tangential velocity profile and $E_{\|}$is the applied tangential electric field. The zeta potential $\zeta$ is defined as the solvent flow infinitely far away from the surface [24, 25, 33] $\zeta=-\left.\eta u\right|_{z \rightarrow \infty} / \varepsilon \varepsilon_{0} E_{\|}$. Combining Eqs. (2) and (4), and using the boundary conditions $d u /\left.d z\right|_{z \rightarrow \infty}=0$ and $\left.u\right|_{z=0}=0$, the zeta potential follows as

$$
\zeta=-\frac{\eta}{\varepsilon} \int_{0}^{\infty} \frac{\varepsilon_{\perp}(z)}{\eta_{\perp}(z)} \frac{d \psi}{d z} d z .
$$

We approximate the viscosity profile by a box function,

$$
\eta_{\perp}(z)= \begin{cases}\eta_{\text {int }} & \text { for } 0<z<z^{*} \\ \eta & \text { for } z>z^{*}\end{cases}
$$

where $\eta_{\text {int }}$ is the viscosity in the interfacial layer [see Fig. 2(a)]. The width of the viscous interfacial layer generally differs from the dielectric one [24, 25], depending on the assumed values of the interfacial dielectric constant $\varepsilon_{\text {int }}$ and viscosity $\eta_{\text {int }}$. For analytical tractability, we use the same width for both, allowing $\varepsilon_{\text {int }}$ and $\eta_{\text {int }}$ to vary.

Clearly, the ion distributions in the interfacial layer are only well reproduced when using the full dielectric profile. Our approach is valid, nevertheless, because we calculate the potential difference and the velocity far away 

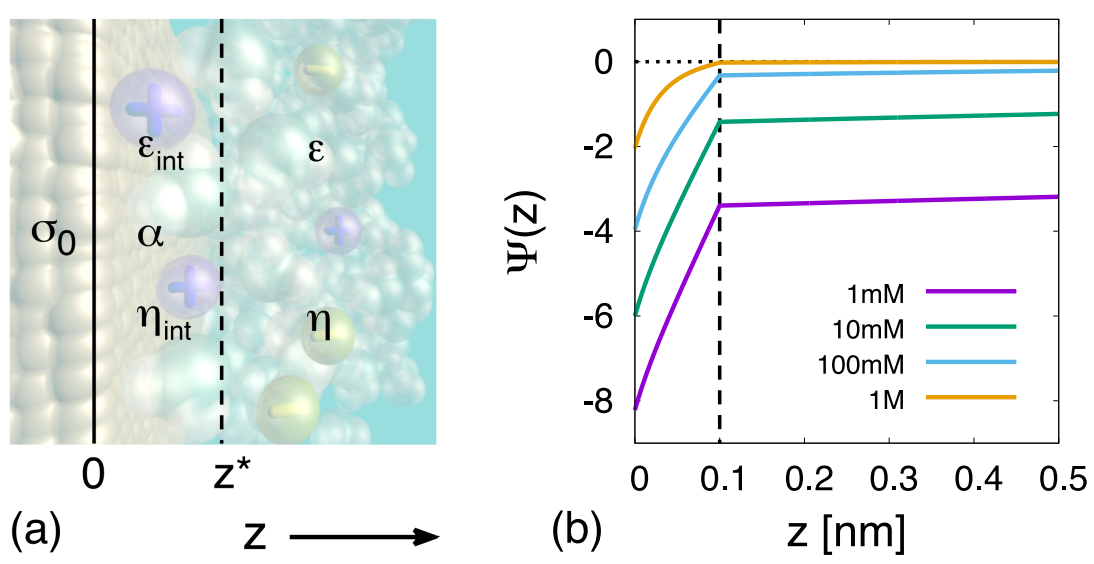

Figure 2: (a) Illustration of the model of the interfacial layer with width $z^{*}$, which has a dielectric constant $\varepsilon_{\text {int }}$, additional chemical potential $k_{\mathrm{B}} T \alpha$, and viscosity $\eta_{\text {int }}$. The surface has a bare surface charge density of $\sigma_{0}$. The region of $z>z^{*}$ has a dielectric constant $\varepsilon$ and viscosity $\eta$. (b) The nondimensional electrostatic potential $\Psi(z)$ for different bulk salt concentrations (monovalent) and $\sigma_{0}=0.1 \mathrm{e} / \mathrm{nm}^{2}$. We set $\varepsilon=78$ [35], $T=298 \mathrm{~K}$, $z^{*}=0.1 \mathrm{~nm}$ (broken line), $\varepsilon_{\mathrm{int}}=1$, and $\alpha=0.0$.

from the surface. In other words, we define the interfacial dielectric and viscous profile such that those quantities can be reproduced by continuum modeling. It has been extensively verified that this box-based continuum model effectively reflects the complex subnanometer interfacial structure well $[22,23,24,25]$. It is this proven correspondence that allows us to use the analytically tractable model presented here to reverse the calculation, and to extract the effective microscopic parameters from macroscopic experiments without relying on MD simulations and numerical calculations.

From Eqs. (3), (5) and (6) the zeta potential follows as [40]

$$
\zeta=\gamma \psi_{0}+(1-\gamma) \psi^{*},
$$

using the surface potential $\psi_{0}=\psi(0)$ and the interfacial-boundary potential $\psi^{*}=\psi\left(z^{*}\right)$, and defining $\gamma=\varepsilon_{\text {int }} \eta / \varepsilon \eta_{\text {int }}$. Based on the Grahame equation, the electrokinetic surface charge density is defined as [24,25]

$$
\sigma_{\mathrm{ek}}=\sqrt{8 \varepsilon \varepsilon_{0} k_{\mathrm{B}} T c_{0}} \sinh \left(\frac{e \zeta}{2 k_{\mathrm{B}} T}\right) .
$$


For homogeneous systems, in which $\eta_{\text {int }}=\eta$ and $\varepsilon_{\text {int }}=\varepsilon$, we obtain $\sigma_{\text {ek }}=\sigma_{0}$. In other words, deviations from $\sigma_{\mathrm{ek}}=\sigma_{0}$ are a measure for interfacial effects.

Exact solution. - Eq. (2) leads to

$$
\frac{d^{2} \Psi}{d z^{2}}= \begin{cases}\left(\varepsilon / \varepsilon_{\mathrm{int}} \mathrm{e}^{\alpha}\right) \kappa^{2} \sinh \Psi & \text { for } 0<z<z^{*} \\ \kappa^{2} \sinh \Psi & \text { for } z>z^{*}\end{cases}
$$

where $\kappa^{-1}$ is the Debye length defined via $\kappa^{2}=2 e^{2} c_{0} / \varepsilon \varepsilon_{0} k_{\mathrm{B}} T$. For $z>z^{*}$, the general solution of Eq. (9) which satisfies the boundary condition at $z \rightarrow \infty$, is given in terms of $\Psi^{*}=\Psi\left(z^{*}\right)$ as

$$
\Psi(z)=2 \ln \frac{1+\mathrm{e}^{-\left(z-z^{*}\right) \kappa} \tanh \left(\Psi^{*} / 4\right)}{1-\mathrm{e}^{-\left(z-z^{*}\right) \kappa} \tanh \left(\Psi^{*} / 4\right)} \text { for } z>z^{*}
$$

Next, we solve Eq. (9) in the range of $0<z<z^{*}$. Eq. (9) is symmetric for cations and anions, so we restrict our discussion to a negatively charged surface. The analytical solution of Eq. (9) for $0<z<z^{*}$ is $[41,42]$

$$
\Psi(z)=\left\{\begin{array}{c}
-2 \operatorname{arctanh}\left[\operatorname{cn}\left(-\kappa \sqrt{\varepsilon / \varepsilon_{\text {int }} \mathrm{e}^{\alpha}}\left(z-z^{*}\right)-F\left(\arccos \left[-\tanh \left(\Psi^{*} / 2\right)\right] \mid 1-p\right) \mid 1-p\right)\right], \\
\text { for } 0 \leq p \leq 1, \\
-2 \operatorname{arctanh}\left[\operatorname{sn}\left(-\sqrt{p} \kappa \sqrt{\varepsilon / \varepsilon_{\text {int }} \mathrm{e}^{\alpha}}\left(z-z^{*}\right)+F\left(\arcsin \left[-\tanh \left(\Psi^{*} / 2\right)\right] \mid 1-p^{-1}\right) \mid 1-p^{-1}\right)\right], \\
\text { for } 1<p,
\end{array}\right.
$$

where $\mathrm{cn}(x \mid m)$ and $\operatorname{sn}(x \mid m)$ are Jacobian elliptic functions [42], $F(\varphi \mid m)$ is the elliptic integral of the first kind [42], and $p$ is an integration constant which satisfies the relation $p=\left[\left(\mathrm{e}^{\alpha} \varepsilon / \varepsilon_{\text {int }}\right)-1\right]\left(\cosh \Psi^{*}-1\right) / 2$. The boundary condition at $z=0$ leads to

$$
-\frac{e \sigma_{0}}{\varepsilon_{\mathrm{int}} \varepsilon_{0} k_{\mathrm{B}} T}=\left\{\begin{array}{c}
-2 \kappa \sqrt{\varepsilon / \varepsilon_{\mathrm{int}} \mathrm{e}^{\alpha}} \mathrm{ds}\left(\kappa \sqrt{\varepsilon / \varepsilon_{\mathrm{int}} \mathrm{e}^{\alpha}} z^{*}-F\left(\arccos \left[-\tanh \left(\Psi^{*} / 2\right)\right] \mid 1-p\right) \mid 1-p\right), \\
\text { for } 0 \leq p \leq 1, \\
2 \sqrt{p} \kappa \sqrt{\varepsilon / \varepsilon_{\mathrm{int}} \mathrm{e}^{\alpha}} \mathrm{dc}\left(\sqrt{p} \kappa \sqrt{\varepsilon / \varepsilon_{\mathrm{int}} \mathrm{e}^{\alpha}} z^{*}+F\left(\arcsin \left[-\tanh \left(\Psi^{*} / 2\right)\right] \mid 1-p^{-1}\right) \mid 1-p^{-1}\right), \\
\text { for } 1<p,
\end{array}\right.
$$

where $\operatorname{ds}(x \mid m)$ and $\operatorname{dc}(x \mid m)$ are Jacobian elliptic functions [42]. Fig. 2(b) shows the electrostatic potential profile, eq. (11), for different bulk salt concentrations and $\sigma_{0}=0.1 \mathrm{e} / \mathrm{nm}^{2}$. We use the parameters $\varepsilon=78$ [35], $T=298 \mathrm{~K}, \varepsilon_{\text {int }}=1, z^{*}=0.1 \mathrm{~nm}$, and $\alpha=0$. In the interfacial layer, the potential decays faster than the region of $z>z^{*}$ due to the low dielectric constant. Both $\left|\Psi_{0}\right|$ and $\left|\Psi^{*}\right|$ decrease with increasing salinity. 

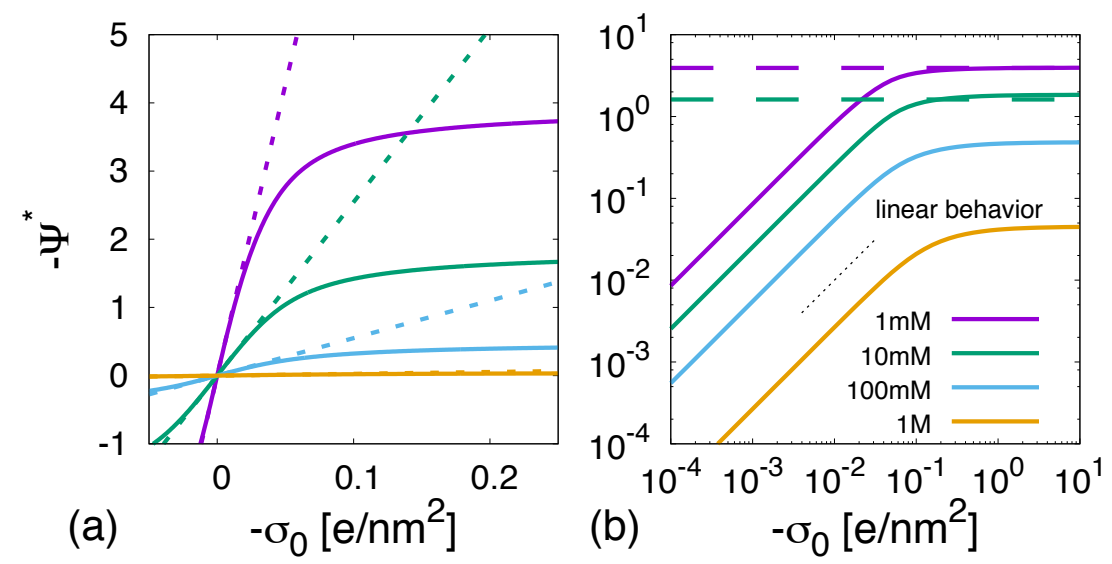

Figure 3: Eq. (12) in terms of the interfacial-boundary potential $\Psi^{*}$ (solid lines). We use the parameters $\varepsilon=78$ [35], $T=298 \mathrm{~K}, \varepsilon_{\text {int }}=1, z^{*}=0.1 \mathrm{~nm}$, and $\alpha=0$. (a) is a linearscale graph, whereas (b) is a double-logarithmic one. Dotted lines in (a) are calculated by linear analysis [33], and broken lines in (b) denote Eq. (13) for $1 \mathrm{mM}$ and $10 \mathrm{mM}$.

From asymptotic analysis of Eqs. (11) and (12) in the limit of strongly charged surfaces and low salinity, the interfacial-boundary potential saturates at the value

$$
\Psi^{*} \approx-\ln \left[\frac{4 \operatorname{arccosh}^{2} \sqrt{\mathrm{e}^{\alpha} \varepsilon / \varepsilon_{\mathrm{int}}}}{\left(\varepsilon / \varepsilon_{\mathrm{int}}-\mathrm{e}^{-\alpha}\right)\left(\varepsilon / \varepsilon_{\mathrm{int}}\right) \kappa^{2} z^{* 2}}\right],
$$

and the surface potential $\Psi_{0}=\Psi(0)$ becomes

$$
\Psi_{0} \approx-\ln \left[\frac{\mathrm{e}^{\alpha} \varepsilon_{\mathrm{int}}}{\kappa^{2} \varepsilon}\left(\frac{e \sigma_{0}}{\varepsilon_{\mathrm{int}} \varepsilon_{0} k_{\mathrm{B}} T}\right)^{2}\right] .
$$

Substituting Eqs. (13) and (14) into Eq. (7), and substituting Eq. (7) into Eq. (8) leads to Eq. (1). Fig. 3 shows the interfacial-boundary potential $\Psi^{*}$ for different salt concentrations as a function of the bare surface charge density $\sigma_{0}$. In Fig. 3(a), the solid lines denote Eq. (12), and the dotted lines denote the linearized solution [33] for parameters $\varepsilon=78$ [35], $T=298 \mathrm{~K}, \varepsilon_{\mathrm{int}}=1, z^{*}=0.1 \mathrm{~nm}$, and $\alpha=0$. For weakly charged surfaces, $\Psi^{*}$ increases linearly with the surface charge density. However, above a specific $\sigma_{0}$ depending on the salinity, $\Psi^{*}$ shows a nonlinear dependence. Fig. 3(b) (double-logarithmic) clearly shows the saturation of $\Psi^{*}$ in the limit of strongly charged surfaces. Horizontal broken lines indicate Eq. (13). The saturation of $\Psi^{*}$ is not unique for this modified PB equation, but also occurs 

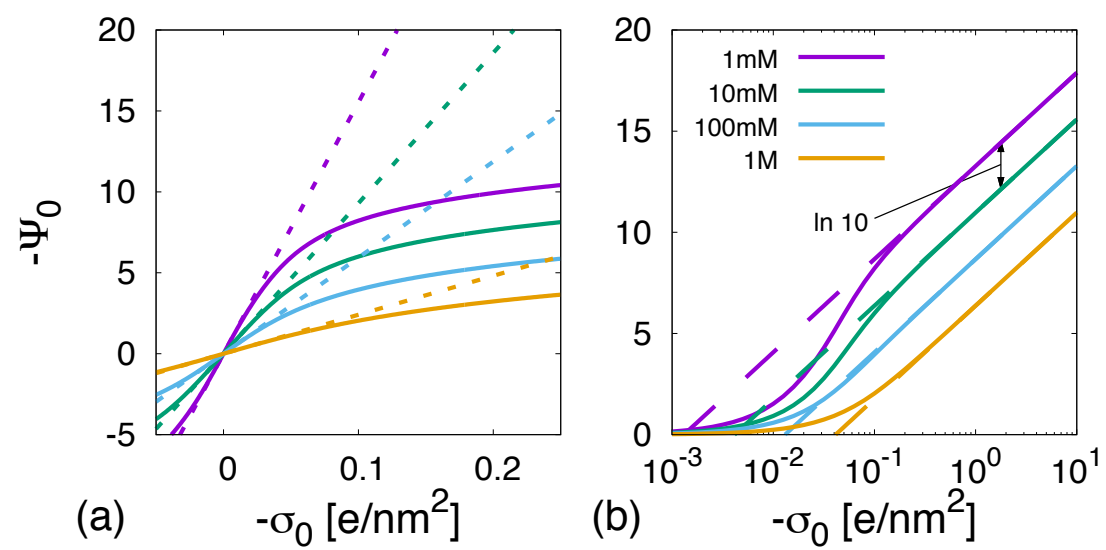

Figure 4: The surface potential $\Psi_{0}$ as a function of the bare surface charge density $\sigma_{0}$ (solid lines). We use the parameters $\varepsilon=78$ [35], $T=298 \mathrm{~K}, \varepsilon_{\mathrm{int}}=1, z^{*}=0.1 \mathrm{~nm}$, and $\alpha=0$. (a) is a linear-scale graph, whereas (b) is a semi-logarithmic one. Dotted lines in (a) are calculated by linear analysis [33], and broken lines in (b) denote Eq. (14).

for a homogeneous dielectric constant [2]. Fig. 4 shows the surface potential $\Psi_{0}$ at a given salt concentration. In Fig. 4(a) (linear scale), the solid lines denote the numerical result obtained by solving Eqs. (11) and (12), and the dotted lines denote the linearized solution [33]. Broken lines in Fig. 4(b) (semi-logarithmic scale) indicate the dependence $\left|\Psi_{0}\right| \sim \ln \left(\sigma_{0}^{2} / c_{0}\right)$ for large $\left|\sigma_{0}\right|$, which follows from Eq. (14).

Summary and conclusions. - We derive an analytical expression for the electrokinetic surface charge density $\sigma_{\text {ek }}$ using a universal model of the structure of the interfacial layer. The model consists of box profiles for the viscosity, the dielectric constant and the specific ion adsorption. In the limit of strongly charged surfaces and low salinity, $\left|\sigma_{\text {ek }}\right|$ increases as a function of the bare surface charge density as a power law with nonuniversal exponent $\gamma=\varepsilon_{\text {int }} \eta / \varepsilon \eta_{\text {int }}$. The exponent is larger for hydrophobic surfaces (where $\eta_{\text {int }}<\eta$ ) than for hydrophilic surfaces (where $\eta_{\text {int }}>\eta$ ), in agreement with experimental data. Fitting the analytical power law to experimental data of $\sigma_{\text {ek }}$ as a function of the bare surface charge density $\sigma_{0}$ gives direct access to the interfacial quantities $z^{*}, \varepsilon_{\text {int }}, \alpha$ and in particular the elusive effective interfacial viscosity $\eta_{\text {int }}$. Therefore, our analytical expression provides a novel method to extract the subnanometer-scale structure of the interfacial layer directly from conventional macroscopic experiments. As the available experimental data is limited and dates back to mid last century, we hope that our 
present results will stimulate new experimental studies.

\section{Acknowledgements}

YU was supported by Grant-in-Aid for JSPS Fellows 13J00063 and 16J00042.

[1] S. S. Dukhin and B. V. Derjaguin, Surface and Colloid Science, ed. E. Matijrvic, vol. 7, Wiley, New York, (1974).

[2] W. B. Russel, D. A. Saville, and W. R. Schowalter, Colloidal Dispersions, Cambridge University Press, Cambridge, (1989).

[3] J. Lyklema, Fundamentals of Interface and Colloid Science, vol.3, Academic Press, London (1995).

[4] L. Joly, C. Ybert, E. Trizac, and L. Bocquet, Phys. Rev. Lett. 93 (2004) 257805.

[5] L. Joly, C. Ybert, E. Trizac, and L. Bocquet, J. Chem. Phys. 125 (2006) 204716.

[6] A. Ajdari and L. Bocquet, Phys. Rev. Lett. 96 (2006) 186102.

[7] D. M. Huang, C. Cottin-Bizonne, C. Ybert, and L. Bocquet, Phys. Rev. Lett. 98 (2007) 177801.

[8] D. M. Huang, C. Cottin-Bizonne, C. Ybert, and L. Bocquet, Langmuir 24 (2008) 1442-1450.

[9] C. I. Bouzigues, P. Tabeling, and L. Bocquet, Phys. Rev. Lett. 101 (2008) 114503.

[10] O. Bonhomme, O. Liot, A.-L. Biance, and L. Bocquet, Phys. Rev. Lett. 110 (2013) 054502.

[11] L. Joly, F. Detcheverry, and A.-L. Biance, Phys. Rev. Lett. 113 (2014) 088301.

[12] S. R. Maduar, A. V. Belyaev, V. Lobaskin, and O. I. Vinogradova, Phys. Rev. Lett. 114 (2015) 118301. 
[13] K. Kim, Y. Nakayama, and R. Yamamoto, Phys. Rev. Lett. 96 (2006) 208302.

[14] V. Lobaskin, B. Dünweg, M. Medebach, T. Palberg, and C. Holm, Phys. Rev. Lett. 98 (2007) 176105.

[15] G. Giupponi and I. Pagonabarraga, Phys. Rev. Lett. 106 (2011) 248304.

[16] M. S. Kilic, M. Z. Bazant, and A. Ajdari, Phys. Rev. E 75 (2007) 021502 .

[17] M. Z. Bazant, B. D. Storey, and A. A. Kornyshev, Phys. Rev. Lett. 106 (2011) 046102.

[18] A. Siria, P. Poncharal, A.-L. Biance, R. Fulcrand, X. Blase, S. T. Purcell, and L. Bocquet, Nature 494 (2013) 455-458.

[19] C. Sendner, D. Horinek, L. Bocquet, and R. R. Netz, Langmuir 25 (2009) 10768-10781.

[20] J.-L. Barrat and L. Bocquet, Phys. Rev Lett. 82 (1999) 4671-4674.

[21] D. M. Huang, C. Sendner, D. Horinek, R. R. Netz, and L. Bocquet, Phys. Rev. Lett. 101 (2008) 226101.

[22] D. J. Bonthuis, S. Gekle and R. R. Netz, Phys. Rev. Lett. 107 (2011) 166102.

[23] D. J. Bonthuis, S. Gekle and R. R. Netz, Langmuir 28 (2012) 76797694.

[24] D. J. Bonthuis, R. R. Netz, Langmuir 28 (2012) 16049-16059.

[25] D. J. Bonthuis and R. R. Netz, J. Phys. Chem. B 117 (2013) 1139711413.

[26] K. Takae and A. Onuki, J. Chem. Phys. 143 (2015) 154503.

[27] Y. Zhu and S. Granick, Phys. Rev. Lett. 87 (2001) 096104.

[28] O. I. Vinogradova and G. E. Yakubov, Langmuir 19 (2003) 1227-1234. 
[29] C. Cottin-Bizonne, B. Cross, A. Steinberger and E. Charlaix, Phys. Rev. Lett. 94 (2005) 056102.

[30] J. Lyklema, J. T. G. Overbeek, J. Colloid Sci. 16 (1961) 501-512.

[31] J. Lyklema, Colloid Surf. A 92 (1994) 41-49.

[32] J. Lyklema, Curr. Opin. Colloid Interface Sci. 15 (2010) 125-130.

[33] D. J. Bonthuis, Y. Uematsu, and R. R. Netz, Phil. Trans. R. Soc. A 374 (2016) 20150033.

[34] O. Stern, Zeitschrift für Elektrochemie, 30 (1924) 508.

[35] B. B. Owen, R. C. Miller, C. E. Milner, and H. L. Cogan, J. Phys. Chem. 65 (1961) 2065.

[36] J. J. Bikerman, Philos. Mag. 33 (1942) 384-397.

[37] M. Eigen and E. Wicke, J. Phys. Chem. 58 (1954) 702-714.

[38] I. Borukhov, D. Andelman, and H. Orland, Phys. Rev. Lett. 79 (1997) 435-438.

[39] I. Borukhov, D. Andelman, and H. Orland, Electrochim. Acta. 46 (2000) 221-229.

[40] Y. Uematsu and T. Araki, J. Chem. Phys. 139 (2013) 094901.

[41] S. H. Behrens and M. Borkovec, Phys. Rev. E 60 (1999) 7040-7048.

[42] M. Abramowitz and I. A. Stegun, Handbook of Mathematical Functions, 9th ed. Dover Publications, New York, (1972). 